\title{
Evaporation-Directed Crack-Patterning of Metal-Organic Framework Colloidal Films and their Application as Photonic Sensors
}

\author{
Olivier Dalstein, ${ }^{[a]}$ Effrosyni Gkaniatsou, ${ }^{[b]}$ Clemence Sicard, ${ }^{[b]}$ Ozlem Sel, ${ }^{[c]}$ Hubert Perrot, ${ }^{[c]}$ Christian \\ Serre, ${ }^{[d]}$ Cédric Boissière, ${ }^{[a]}$ Marco Faustini*[a]
}

\begin{abstract}
Herein, a straightforward crack-patterning method is reported allowing the direct formation of periodic cracks in MetalOrganic Framework nanoparticles films during dip-coating deposition. The crack propagation and periodicity can be easily tailored by controlling the evaporation front and the withdrawal speed. Several MOF patterned films can be fabricated on large surfaces and on several substrates (flat, curved or flexible) including the inner surface of a tube, not achievable by other lithographic techniques. We demonstrate that the periodic cracked arrays diffract light and, thanks to the MOF sorption properties, photonic vapor sensors are fabricated A new concept of "in-tube", MOF-based diffraction grating sensors is proposed with outstanding sensitivity that can be easily tuned "ondemand" as function of the desired detection range.
\end{abstract}

Thanks to their chemical versatility and to their tunable porosity Metal-Organic Frameworks (MOFs) are promising materials for a wide range of applications. In order to fulfill their promises, MOFs need to be processed, shaped and integrated in functional devices. ${ }^{[1]}$ In particular, patterning MOF films could potentially open perspectives for their full utilization in fields ranging from electronics, photonics, microfluidics, water harvesting or separation. [2] For example, in the field of photonics, the combination between the unique properties of MOFs (high porosity, sorption selectivity..) and of periodic structures (light confinement, diffraction, guiding..) could allow the fabrication of a new generation of performing optical sensors. In this context, deposition and patterning of MOF films has become a scientific and technological, interdisciplinary challenge. Several "top-down" approaches based on lithographic methods have been reported to pattern MOF-based films. ${ }^{[3]}$ While providing an excellent control, all these methods present critical drawbacks: they are not easily accessible, they required more than two processing steps and

[a] O. Dalstein, Dr. C. Boissiere, Dr. M. Faustin

Sorbonne Universités, UPMC Univ Paris 06, CNRS, Collège de France, UMR 7574, Chimie de la Matière Condensée de Paris, Paris, France. E-mail: marco.faustini@upmc.fr

[b] E. Gkaniatsou, Dr. C. Sicard

Institut Lavoisier de Versailles, UVSQ, CNRS, Université ParisSaclay, Versailles, France.

[c] Dr. O. Sel, Dr. H. Perrot

Sorbonne Universités, UPMC Univ. Paris 06, CNRS, UMR 8235 , LISE, Paris, France

[d] Dr. C. Serre

Institut des Matériaux Poreux de Paris, FRE 2000 CNRS-ENSESPCI, PSL research university, 75005, Paris, France.

Supporting information for this article is given via a link at the end of the document. they present limitations related to substrate geometry, scalability, versatility and/or cost. Ideally, the fabrication of the future MOFpatterned devices needs to be easy, single-step, cheap, scalable and compatible with various substrates/geometries. Recently, crack-patterning has emerged as an alternative and convenient "bottom-up " method to pattern thin films. ${ }^{[4]}$ In controlled conditions, crack propagation can proceed following straight, oscillatory or spiral-like paths ${ }^{[5]}$ and/or it can be guided by micronotches (made by lithographic techniques) toward complex patterns and devices. ${ }^{\left[{ }^{[6]}\right.}$ Alternatively, as observed in mud's cracks, Allain and Limat ${ }^{[7]}$ demonstrated that controlled drying of colloidal droplets resulted in the formation of parallel periodic cracks oriented by the evaporation front-line. This approach was recently proposed as microfabrication too ${ }^{[8]}$ yet by using conventional colloidal materials (such as silica or polystyrene beads). In addition, real applicative examples of those colloidal crackpatterned surfaces are still missing.

Herein we demonstrate that Metal-Organic Framework colloidal films can be crack-patterned during dip-coating deposition where the crack propagation is oriented by the evaporation front. This versatile method allows preparation of periodic crack arrays with tunable periodicity, on large surface and on substrates having various chemical nature and geometry (including the inner surface of a tube) not achievable with other lithographic techniques. ${ }^{[9]}$ For the first time, we show that arrays of periodic cracks diffract lights and can be used as photonic platform. We also propose a new concept of "on-demand" optical sensors, based on MOF diffraction gratings, with outstanding sensitivity, easily adjustable as function of the desired detection range.

Due to its outstanding chemical stability and its very high accessible porosity, MIL-101(Cr) colloids were chosen as a first case of study. ${ }^{[10]}$ The MIL-101(Cr) nanoparticles, made through a microwave assisted hydrothermal synthesis, having average size of around $25 \mathrm{~nm}$ (from SEM) were characterized by powder x-ray diffraction (PXRD) (Figure S1). Figure 1 (a) schemes the dipcoated assisted crack patterning approach. In the process, the substrate is first dipped into an ethanolic MIL-101(Cr) colloidal solution and withdrawn at low speed. Since the deposition process strongly depends on the atmosphere, the humidity into the chamber was fixed at values $<10 \% \mathrm{RH}$. During dip coating, the evaporation-directed crack formation in the MIL-101 films was recorded by a homemade optical microscopy set-up (Figure S2); a characteristic micrograph and the illustration of the possible spatio-temporal evolution of the evaporating system is shown in Figure 1 (b). Three characteristic zones can be identified. Just above the triple line meniscus, in Zone I, the colloidal MIL-101 nanoparticles form a "wet" colloidal gel made of percolating particles and filled with the solvent. Internal stresses start to 
accumulate because of the shrinkage provoked by solvent the partial delamination at cell's edge as opposed to the adhesion

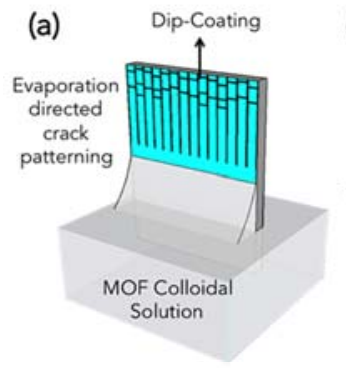

(b)
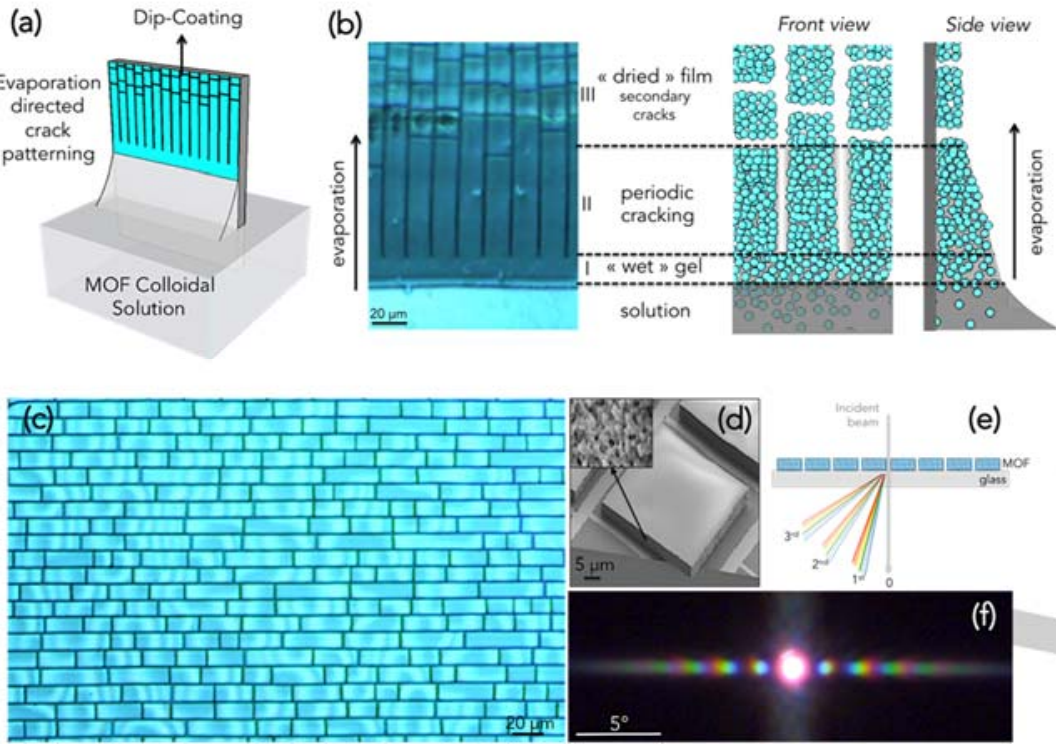

Figure 1 (a) Illustration of the dip-coating assisted crack-pattering process; (b) optical micrograph (and illustration) of the evaporation directed crack formation and propagation; (c) optical micrograph of a crack-patterned MIL-101(Cr) film; (d) SEM photograph of a cracked cell; (e) schematic view of the diffraction grating performance of the MOF crack-patterned film visualized by Conoscopic

Microscopy (f).

removing. In Zone II, regularly spaced cracks appear to release the evaporation-induced stress and then propagate perpendicularly to the drying line. At low speed $\left(<0.5 \mathrm{mms}^{-1}\right)$, the pace of crack propagation is the same than the withdrawal speed. Zone III is located above the drying line where the complete solvent evaporation induces strong capillary stresses released by the formation of perpendicular random secondary cracks. The dried film is thus composed by an oriented, regularly spaced, brick-like micropatterns. The exact mechanism of the evaporation-directed crack propagation, observed in Zone II, was first described for colloidal silica by Allain and Limat as a result of two opposite phenomena. ${ }^{[7]}$ Formation of periodic cracks results from competition between stress relaxation (due to crack opening) and accumulation (due to evaporation through the newly opened crack surface). The combination of these two contributions yields to a maximum in the variation of the global stress, located at a precise distance from the first crack, where the next crack will form. As a next demonstration of this concept, the same experiment was performed with ZIF-8 colloidal solution having average size of $32 \mathrm{~nm}$ (from SEM) and characterized by PXRD (Figure S3); as shown in Figure S4, the same evaporation-directed crack patterning mechanism was observed, confirming the applicability of the approach on several colloidal MOFs with different surface chemistry and polar characters. An optical micrograph of a crack-patterned MIL-101 film obtained by dip coating at $0.03 \mathrm{mms}^{-1}$ on Silicon wafer is shown in Figure 1(c). The microstructure of each cracked cell is displayed in the SEM micrograph of Figure 1 (d) while the inset highlights that the film is made of packed MIL-101 nanoparticles (Figure S5). SEM and AFM analysis in Figure S5 reveals a slight thickness increase $(<12 \%)$ at the edge of each rectangular cell; indeed a slight buckling is often observed due to stress release caused by at the cell's center. ${ }^{[11]}$ Due to this high degree of order of the periodic structure, the crackpatterned MOF film acts as efficient diffraction gratings which splits and diffracts light into several beams travelling in different directions as shown in Figure 1(e). This dispersive capability of a crack-patterned MIL-101 film coated on glass (periodicity $=16 \mu \mathrm{m}$ ) was proved by Conoscopic Imaging in transmission mode performed using a microscope equipped with a Bertrand lens. ${ }^{[12]}$ The presence of several rainbow-colored patterns, shown in Figure 1(f), indicates that light is angularly diffracted and split-up into several diffraction orders.

The crack periodicity can be advantageously tuned by controlling the withdrawal speed $(u)$ during dip-coating. This correlation is reported in Figure 2(a) for MIL-101(Cr) on silicon where two main tendencies are observed. At high dip rate ("draining regime), fast solvent evaporation induces not-organized, mud-like cracked networks. Oriented periodic cracks are obtained only at low speeds $\left(<1 \mathrm{mms}^{-1}\right)$ in the so-called "capillary regime". ${ }^{[13]}$ The periodicity of the parallel crack can be tuned from 47 down to $2 \mu \mathrm{m}$ by increasing the withdrawal speed from 0.02 to $1 \mathrm{mms}^{-1}$ respectively (at room temperature). According with the literature $^{[7-8]}$ the crack periodicity is proportional to the film's thickness; this correlation was verified here, with a periodicity/thickness ratio equal to 3.5 for deposition on silicon wafers (Figure S6). This tunability can be observed in the photograph in Figure 2(b) in which films dip-coated at increasing speeds (from 0.01 to $0.05 \mathrm{mms}^{-1}$ ) exhibit different iridescence colors due to variation in crack periodicity/thickness. The effect of the wetting properties of the substrate was investigated by deposition of MOFs on silicon wafer coated sol-gel based hybrid films obtained from different TEOS/MTEOS ratios (Figure S7); ${ }^{[14]}$ similar results in term of periodicity vs speed were obtained by dip-coating onto surfaces characterized by contact angle in the $30-80^{\circ}$ range (Figure S8). This versatility allows implementation of the crack-patterning on different supports including flexible
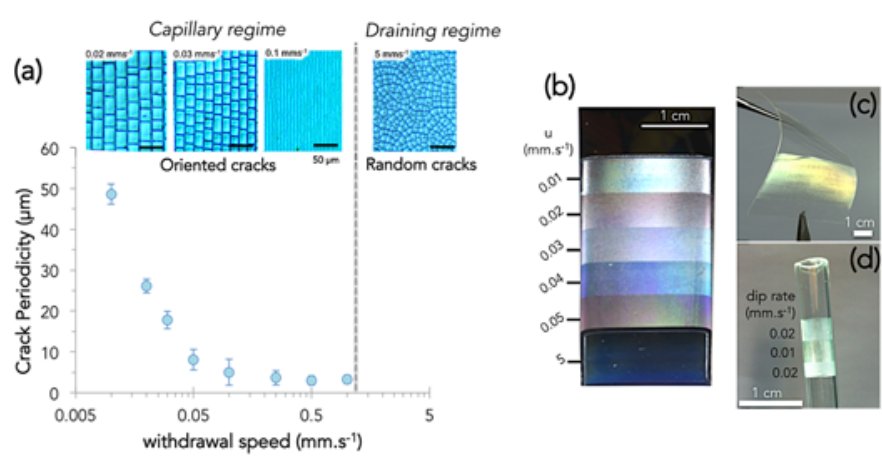

Figure 2 (a) Plot of crack periodicity as function of the dip-coating withdrawal speed; photographs of crack-patterned diffraction gratings on (b) silicon, (c) flexible substrate and (d) inner and outer surface of a glass tube. 
plastic substrate (polypropylene) Figure 2(c) or even not-flat, curved substrate. Importantly, this approach based on periodic cracks, allows micropattering (and formation of diffraction gratings) on the inner and outer surfaces of a glass tube, Figure 2(d), not achievable with other conventional pattering approaches. As mentioned above, MOF films characterized by periodic array of cracks acts as diffraction grating. Taking advantage of the high porosity and of the selective sorption properties of MIL-101(Cr) and on the versatility of the crack-pattering process, a new kind of diffraction grating vapor sensors was developed.

The signal transduction relies on the change of refractive index $\Delta \mathrm{n}$ in response to the analyte adsorption into the porosity. ${ }^{[15],[10 a \text {, }}$ 16] Previous works demonstrated that MOFs could be integrated in photonic « colorimetric » vapor sensors such as thin films, [10a, 16] Fabry-Pérot, ${ }^{[17]}$ 1D Bragg stacks, ${ }^{[18]} 3 \mathrm{D}$ colloidal crystals ${ }^{[19]}$ for which vapor adsorption leads to a shift of a characteristic wavelength $\Delta \lambda$. Recently some of us reported a promising alternative approach based on ZIF-8-based 2D photonic structures whose optical output consisted in a variation of diffracted light intensity (instead of color shift). ${ }^{[3 \mathrm{~d}]}$ The detection mode of our crack-patterned sensor is illustrated in Figure 3(a): MIL-101(Cr) diffraction gratings were deposited on transparent glass substrate; the films are irradiated by a monochromatic light beam $(\lambda=640 \mathrm{~nm})$ and the corresponding diffraction pattern intensities are recorded as function of the atmosphere composition $\left(\mathrm{P} / \mathrm{P}_{0}\right)$ by a simple $C C D$ camera (Figure $\left.\mathrm{S} 9\right)$. In this study, we mainly monitored the $1^{\text {st }}$ order diffraction intensity $\left(I_{1}\right)$. Figure 3(b) displays the evolution of the normalized intensity of the $I_{1}$ as function of the IsoPropyl Alcohol (IPA) relative vapor pressure for a crack-patterned sample having thickness and periodicity equal to 3.9 and $23 \mu \mathrm{m}$, respectively. Indeed vapor adsorption into the MIL-101(Cr) pores causes a refractive index increase that impacts the intensity of the diffracted light. However, differently from other optical devices, the trend is not linear. Firstof-all, at low IPA concentrations $\left(P / P_{0}<6 \%\right)$, the device is very sensitive since a great increase in $I_{1}$ up to $164 \%$ is observed; at higher concentration, $I_{1}$ decreases back to low values. This trend can be predicted semi-empirically following models proposed by Whitesides and co-workers ${ }^{[20]}$ for gratings made of arrays of lines. According to the theory in the configuration illustrated in Figure 3(a), a phase difference $(\varphi)$ is created when light passes through the MOF-based crack-patterns. The phase difference depends on the light wavelength $\lambda$, on the thickness $h$, and on the refractive index contrast between the colloidal MOF film ( $n_{\text {MOF }}$ ) and the atmosphere ( $\left.n_{\text {air }}=1\right)$ and is given in Equation (1):

$$
\varphi=\frac{2 \pi h\left(n_{M O F}-n_{\text {air }}\right)}{\lambda}
$$

The first order diffracted intensity can be deduced from $\varphi$ and is given in Equation (2)

$$
I_{1}=\frac{2(1-\cos \varphi)}{\pi^{2}}
$$

Since both $\mathrm{n}_{\mathrm{MOF}}$ and $\mathrm{h}$ vary with IPA P/P $\mathrm{P}_{0}$, this correlation was accessed by in-situ Environmental Ellipsometry Porosimetry (EEP) on a thin, not-cracked, optical MIL-101 film as shown in
Figure 3(c). Increase of $P / P_{0}$ results in a raise of $n($ at $640 \mathrm{~nm}$ ) and a slight contraction of the colloidal films. This sorption behavior of MIL-101(Cr) was globally confirmed by the mass uptake of IPA measured by Quartz Crystal Microbalance (QCM) in controlled atmosphere (Figure S10). The evolution of $n$ vs $P / P_{0}$ is characterized by two steps at $13 \%$ and $20 \% \mathrm{P} / \mathrm{P}_{0} \%$ indicating capillary condensation in the 2.9 and $3.4 \mathrm{~nm}$ mesopores of MOF,
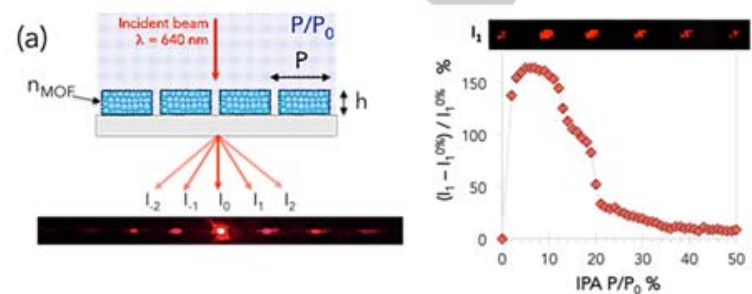

(b)
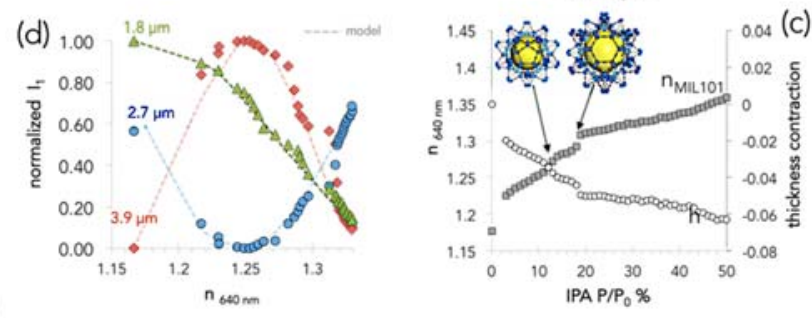

Figure 3 (a) Schematic view of the detection experiment on MOF (MIL101(Cr)) diffraction gratings; (b) plot of normalized $\mathrm{I}_{1}$ versus IPA P/P $\%$ (thickness $=3.9 \mu \mathrm{m}$ ) ; (c) evolution of the refractive index and thickness versus IPA P/P $\%$ determined by in-situ ellipsometry; (d) experimental and predicted evolution of normalized $I_{1}$ vs refractive index for diffraction gratings having different thickness

respectively. The predicted and experimental normalized $l_{1}$ as function of refractive index variation was obtained by adding the experimental $n$ and $h$ as function of $P / P_{0}$ (obtained by EEP) into equation (1) and (2). Figure 3(d) shows the tendencies for 3 crackpatterned films with $\mathrm{h}=1.8,2.7$ and $3.9 \mu \mathrm{m}$ (thicknesses measured by AFM); the model curves globally correlate the experimental tendencies. Slight deviations are observed for relative high $P / P_{0}$ for $n$ comprised between 1.27 and 1.32 probably due to film structural modification occurring during capillary condensation. As a consequence, this study indicates that, knowing $\mathrm{n}$ and $\mathrm{h}$ evolutions of the MOF material, the sensor response can be predicted and tuned by thickness selection. This property of diffraction gratings allows fabrication of sensors with "on-demand", enhanced sensitivity depending on the detection range. Figure 4 reports a real case of application of a MIL-101(Cr) crack-patterned sensor (i) exhibiting high sensitivity at low vapor concentration, (ii) compatible with CCD camera and smart-phone transduction and (iii) integrated in the inner surface of a tube for continuous airflow monitoring. The design of the crack-patterned sensor was first guided by predictive model; its thickness was set in order to maximize the sensitivity for ultra-low refractive index variation (<0.02), corresponding to adsorption at ultra-low vapor pressure (typically $<1 \% \mathrm{P} / \mathrm{P}_{0}$ ). Figure $4(\mathrm{a})$ shows the plots of the predicted $I_{1}$ vs $n_{640}$ sinusoidal curves for three thicknesses and calculated from to equation (1) and (2). The corresponding optical sensitivities (slopes $\mathrm{dl}_{1} / \mathrm{dn}_{640}$ ), shown in Figure $\mathbf{4}(\mathbf{b})$, indicates that the $4.5 \mu \mathrm{m}$-thick cracked-patterned sensor exhibits the highest (and constant) sensitivity in the low-vapor pressure range. 
Starting from these results, crack-patterned MIL-101(Cr) film having thickness equal to $4.5 \mu \mathrm{m}$ (measured by $\mathrm{AFM}$ ) was deposited onto the inner surface of a tube with squared section. The photograph of the macroscopic diffracting zone and optical micrograph of a crack-patterned area are shown in Figure 4(c) and in Figure S11. The performances of the "in-tube" MIL-101(Cr) diffracting sensor were tested in presence of low concentration of toluene vapors (in air) by monitoring the evolution of $\mathrm{I}_{1}$ with a CCD camera as illustrated in Figure 4(d) and plotted in Figure 4(e). In agreement with the prediction, increase in toluene $P / P_{0}$ in the low vapor pressure range results in a pseudo-linear increase of $I_{1}$. Importantly, a slight increase of toluene concentration $(<1.2 \%$ $\mathrm{P} / \mathrm{P}_{0}$ ) leads to a tremendous $\mathrm{I}_{1}$ change (up to $60 \%$ ). The chemical sensitivity of this device can be quantified in $5500 \%$. $\left(\mathrm{P} / \mathrm{P}_{0}\right)^{-1}$ (slope of the $I_{1} /$ concentration curve) allowing toluene detection well below the permissible exposure limits $(200 \mathrm{ppm})$ that can be easily recorded by a simple CCD camera. The sensitivity performance of the crack-patterned sensor has been compared through optical simulation to MIL-101 based Fabry-Perot and Bragg-Mirror sensors by adapting geometry and structural parameters from literature data ${ }^{[17,18 c]}$. As detailed in Figure S12 and Figure S13, in this configuration, the crack-patterned sensor shows the best sensitivity that is comparable (and even higher) to the Bragg-Mirror system; this is remarkable considering that a Bragg-Mirror requires 6 steps of deposition of 2 different materials while crack-patterning can be realized in a single step. A further detection experiment was performed with crack-patterned sensor with a different material (ZIF-8), contaminant (EtOH) and detection mode (0 order diffraction). As shown in Figure S14, the detection is effective and reversible suggesting that the approach based on MOF crack-patterned diffraction gratings sensors can be generalized to several systems. This is important since multimaterials platforms can be envisioned to carry out combinatorial sensing to improve selectivity in presence of mixture of pollutants. ${ }^{[18 c]}$ In conclusion, a dip-coating assisted crackpatterning approach was demonstrated allowing for the first time formation of oriented periodic cracks in MOF films on large surfaces and on several substrates with various shapes (flat, flexible or tubular). The crack arrays act as diffraction gratings with periodicity/thickness that can be easily tuned in the
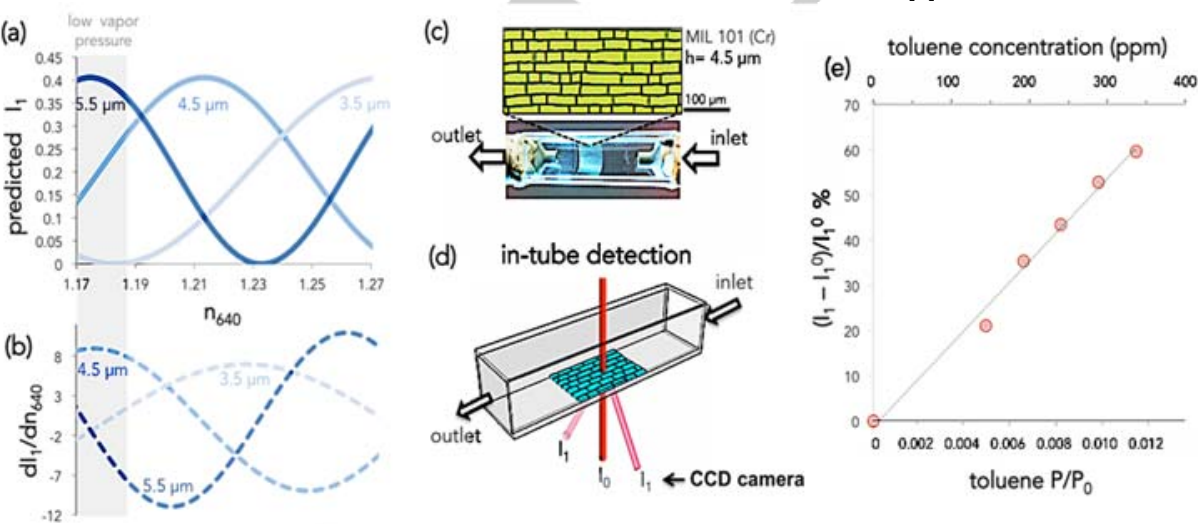

Figure 4 Predicted (a) first order intensity and (b) sensitivity as function of refractive index for diffraction gratings having different thickness. (c) Optical micrographs of MIL-101(Cr) crack patterned film coated into a tube; (d) scheme of the "intube" detection experiment. (e) Plot of normalized $\mathrm{I}_{1}$ as function of the toluene concentration. micrometric range. A new concept of "in-tube", MOF-based diffracting vapor sensors is proposed in which enhanced sensitivity can be tuned as function of the desired detection range. Importantly, due its versatility and low-cost, the MOF crackpattering approach can be further implemented towards integration of MOFs in other domains that requires patterning (electronics, microfluidics, separation and so on). In addition, this "bottom-up" method can be further extended to other nanomaterials (quantum dots, plasmonic particle..) for their direct integration in two-dimensional photonic devices without the limitations of the conventional lithographic techniques.

\section{Experimental Section}

Experimental Details are reported in Supplementary Information

\section{Acknowledgements}

We thank David Montero for his support for electronic microscopy (Institut des Matériaux de Paris Centre FR2482). E. G thanks thank the ANR-11-LABEX-0039 for funding.

Keywords: Crack, Metal-Organic Frameworks, Nanoparticles, Diffraction, Sensing

[1] a G. Maurin, C. Serre, A. Cooper, G. Ferey, Chemical Society Reviews 2017, 46, 3104-3107; b J.-O. Kim, K.-I. Min, H. Noh, D.-H. Kim, S.-Y. Park, D.P. Kim, Angew. Chem. Int. Ed. 2016, 55, 7116-71120.

[2] I. Stassen, N. Burtch, A. Talin, P. Falcaro, M. Allendorf, R. Ameloot Chemical Society Reviews 2017, 46, 3185-3241.

[3] aC. M. Doherty, G. Grenci, R. Ricco, J. I. Mardel, J. Reboul, S. Furukawa, S. Kitagawa, A. J. Hill, P. Falcaro, Adv. Mater. 2013, 25 4701-4705; bG. Lu, O. K. Farha, W. N. Zhang, F. W. Huo, J. T. Hupp, Adv. Mater. 2012, 24, 3970-3974; cB. K. Keitz, C. J. Yu, J. R. Long, R. Ameloot, Angew. Chem.-Int. Edit. 2014, 53, 5561-5565; dO. Dalstein, D. R. Ceratti, C. Boissière, D. Grosso, A Cattoni, M. Faustini, Advanced Functional Materials 2016, 26, 81-90; eP. Falcaro, D. Buso, A. J. Hill, C. M. Doherty, Adv. Mater. 2012, 24 3153-3168.

[4] D. Kang, P. V. Pikhitsa, Y. W. Choi, C. Lee, S. S. Shin, L. Piao, B. Park, K.-Y. Suh, T.-i. Kim, M. Choi, Nature 2014, 516, 222-226.

J. Marthelot, B. Roman, J. Bico, J. Teisseire, D. Dalmas, F. Melo,

[5] Marthelot, B. Roman, J. Bico, J. Teisseire, D. Dalmas, F. Melo,
Physical review letters 2014, 113, 085502. Kim, D. Ha, T. Kim, Nature Communications 2015, 6, 6247. [7] C. Allain, L. Limat, Physical review letters 1995, 74, 2981.

[8] aB. Li, B. Jiang, W. Han, M. He, X. Li, W. Wang, S. W. Hong, M. Byun, S. Lin, Z. Lin, Angewandte Chemie International Edition 2017, 56, 43634363; bW. Han, B. Li, Z. Lin, ACS Nano 2013, 7, 6079-6085.

[9] D. R. Ceratti, B. Louis, $X$ Paquez, M. Faustini, D. Grosso, Adv Mater. 2015, 27, 4958.

[10] aA. Demessence, $P$ Horcajada, C. Serre, C. Boissiere, D. Grosso, C. Sanchez, G. Ferey, Chem. Commun. 2009, 7149-7151; bG. Férey C. Mellot-Draznieks, C. Serre, F. Millange, J. Dutour, S. Surblé, I. Margiolaki, Science 2005, 309, 2040. [11] L. Pauchard, EPL (Europhysics Letters) 2006, 74, 188.

[12] S. N. Fernandes, Y. Geng, S. Vignolini, B. J. Glover, A. C. Trindade, J. P. Canejo, P. L. Almeida, P 
Brogueira, M. H. Godinho, Macromolecular Chemistry and Physics 2013, 214, 25-32

[13]

aM. Faustini, B. Louis, P. A. Albouy, M. Kuemmel, D. Grosso, Journal of Physical Chemistry C 2010, 114, 7637-7645; bM. Faustini, C. Boissière, L. Nicole, D. Grosso, Chemistry of Materials 2013

[14] K. Sumida, K. Liang, J. Reboul, I. A. Ibarra, S. Furukawa, P. Falcaro, Chemistry of Materials 2017, 29, 2626-2645.

W. P. Lustig, S. Mukherjee, N. D. Rudd, A. V. Desai, J. Li, S. K. Ghosh, Chemical Society Reviews 2017, 46, 3242-3285.

[16] A. Demessence, C. Boissiere, D. Grosso, P. Horcajada, C. Serre G. Ferey, G. Soler-Illia, C. Sanchez, Journal of Materials Chemistry 2010, 20, 7676-7681.

[17] G. Lu, J. T. Hupp, Journal of the American Chemical Society 2010 $132,7832-7833$
[18]

F. M. Hinterholzinger, A. Ranft, J. M. Feckl, B. Ruhle, T. Bein, B. V. Lotsch, Journal of Materials Chemistry 2012, 22, 10356-10362 bZ. Hu, C.-a. Tao, F. Wang, X. Zou, J. Wang, Journal of Materials Chemistry C 2015, 3, 211-216; cA. Ranft, F. Niekiel, I. Pavlichenko, N. Stock, B. V. Lotsch, Chemistry of Materials 2015, 27, 1961-1970. G. Lu, O. K. Farha, L. E. Kreno, P. M. Schoenecker, K. S. Walton, R. P. Van Duyne, J. T. Hupp, Adv. Mater. 2011, 23, 4449-+.

aO. J. A. Schueller, D. C. Duffy, J. A. Rogers, S. T. Brittain, G. M Whitesides, Sensors and Actuators A: Physical 1999, 78, 149-159; bB. A. Grzybowski, D. Qin, G. M. Whitesides, Applied optics 1999 38, 2997-3002. 
Entry for the Table of Contents (Please choose one layout)

Layout 1:

\section{COMMUNICATION}

A dip-coating method allowing simultaneous deposition and crackpatterning of Metal-Organic Frameworks colloidal films is demonstrated. The oriented periodic cracks arrays are tunable, act as diffraction gratings and can be fabricated on large surfaces, on several substrates with complex shapes. A new concept of "in-tube", MOF-based diffracting vapor sensors is proposed in which the sensitivity range can be tuned "on-demand".

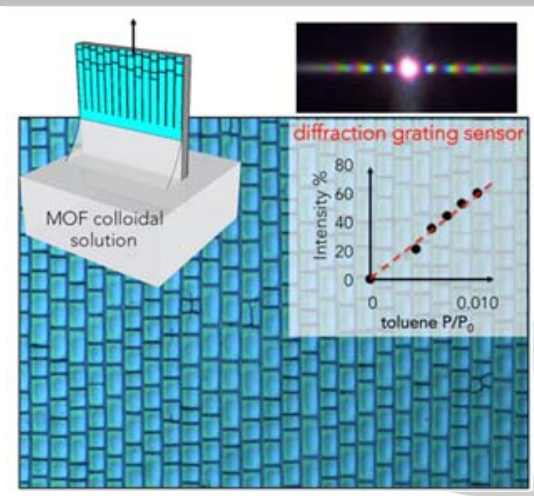

O. Dalstein, E. Gkaniatsou, C. Sicard, O. Sel, H. Perrot, C. Serre, C.

Boissière, M. Faustini *

Page No. - Page No.

Evaporation-Directed Crack-

Patterning of Metal-Organic

Framework Colloidal Films and their

Application as Photonic Sensors 